\title{
Beam Pattern Design Method Based on Particle Swarm Optimization
}

\author{
Yan Rong ${ }^{\text {a)}, \text { Shuqiang Gao }}{ }^{\text {b) }}$ \\ School of Information Engineering, Zhengzhou University, Zhengzhou 450000, China. \\ a)wbt961112@163.com \\ b)736640027@qq.com
}

\begin{abstract}
As a new and emerging intelligent algorithm, although young, but the particle swarm algorithm has a very important role in the development of modern society, so we can do nothing without it, its emergence in the academic community caused Depressed big wave. In this article, we try to give its principle, and of course we will also show you how to design a beam pattern based on it. Obviously, in order to highlight our correctness, we compared this method with the simulated annealing algorithm. We proposed the design of a 20 -element planar array because we will show you the effectiveness of our method. Finally, we can see that using the particle swarm algorithm we get a better beam pattern, which means we get a lower side-lobe.
\end{abstract}

Key words: Genetic Algorithm; Beam-forming; Particle Swarm; Beam Pattern Design.

\section{INTRODUCTION}

We know that the purpose of beam-forming is to receive signals arriving in a specified direction and to suppress interference from other directions to the greatest extent. Its main function is to perform certain spatial processing ie spatial filtering, in order to obtain good anti-noise, Interference and spatial gain of anti-reverberation clutter, and determination of the azimuth and elevation information of the target [1]. Many authors have proposed various ways to optimize the pattern [2]. A measure of the ability to suppress interference is to see if the side lobe of the designed beam pattern is low. [4] It is clear that clutter and all kinds of interference will enter the receiver through the side lobes. If the side lobe of the designed beam pattern is very low, it is of practical value. Therefore, it is very necessary to study how to make the side lobes of the array low.

The simulated annealing algorithm [3] is a random global optimization algorithm [8]. If the energy function is not selected properly, the algorithm will not converge and will cause great trouble for the calculation. The particle swarm algorithm is a parallel algorithm that belongs to swarm intelligence algorithm. It is also called bird foraging algorithm. It is a new optimization algorithm. It is very useful and very good. It has been developed in recent years.

\section{PARTICLE SWARM OPTIMIZATION}

From the simple natural phenomenon of bird foraging, we humans were inspired to discover the particle swarm algorithm. We can imagine such a beautiful scene: a flock of birds is aimlessly looking for the food they want, but there is only one piece of food in this area. However, the birds do not know where the food is, but they know how far away they are from the food. How to plan their own route is the best? The simplest and most effective way we can think of is to search for the area around the bird that is currently closest to food [9] [10] [11]. The following allows me to explain to you some of the considerations of the very powerful algorithm of the particle swarm. The solution to each optimization problem is regarded as a bird [12]. Through iterative methods, we can find a best strategy for birds. The concise and concise optimization process can be shown as follows: 


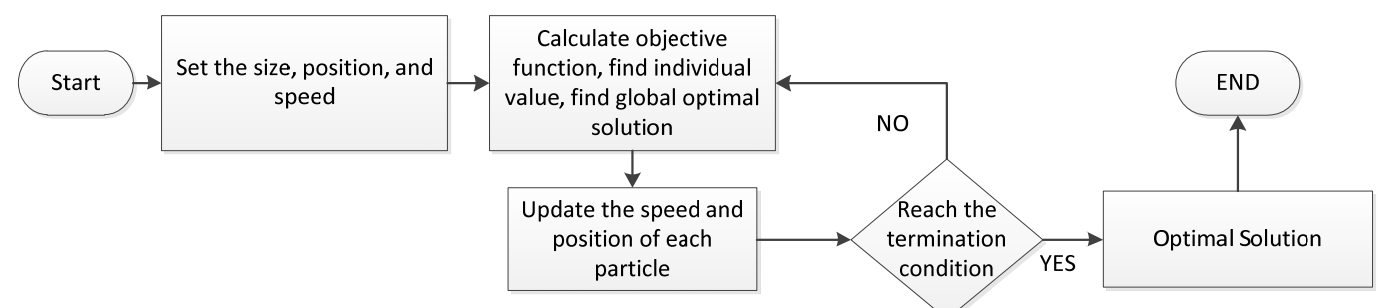

FIGURE 1. The optimization processes

\section{MATHEMATICAL MODEL OF BEAM DESIGN FOR PARTICLE SWARM OPTIMIZATION}

In general, the structural diagram of the beam-former can be as shown in the following figure: There are $\mathrm{N}+1$ per inputs, there are $\mathrm{N}+1$ weighting coefficients, and naturally there are $\mathrm{N}+1$ outputs. We use $\alpha$ instead of the general direction of the signal (because we don't know it yet) and we can use vectors to represent it. That is, the vector of the signal is $X=\left[x_{0}, x_{1}, x_{2},,, x_{N}\right]^{T}$, The vector of weighting coefficients is $W=\left[\omega_{0}, \omega_{1}, \omega_{2},,,, \omega_{N}\right]^{T}$, The total output vector is:

$$
y(\alpha)=W^{T} X
$$

Set the array in the direction of $\alpha$ the maximum output is $y\left(\alpha_{0}\right)$, then use this to do a normalized output. For its modulus, we do a square process. In order to simplify the expression, we perform logarithm processing on it, so that everyone can observe and obtain the beam diagram:

$$
G(\alpha)=10 \lg \frac{|y(\alpha)|^{2}}{\left|y\left(\alpha_{0}\right)\right|^{2}}
$$

Obviously, if we use different weight vector we can get different beam patterns. Assumed that $G_{l}(\alpha)$ Is the beam pattern we want to get, so obviously our task is how to choose a suitable set of weight coefficients so that $G(\alpha)$ Equal to or infinitely close to $G_{l}(\alpha)$

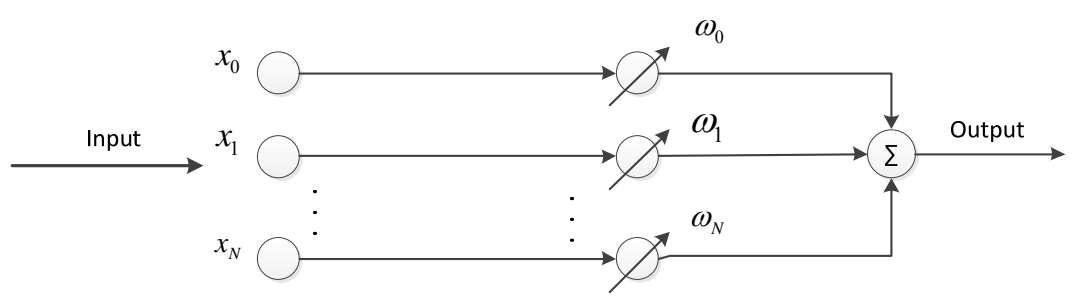

FIGURE 2. Model of beam-forming

Below we apply the particle swarm algorithm to the task of designing beam patterns, introducing a cost function, we minimize the cost function, and we can get the best weight coefficient vector at this time.

The cost function is:

$$
E(W, \alpha)=E\left[\left|G(\alpha)-G_{l}(\alpha)\right|^{2}\right]
$$

In this way, if we can minimize the cost function, then we can solve the problem of optimizing the beam pattern. Smart you can see that what we have to do is to make the cost function small, how can we make it smaller? It is obvious that choosing the right coefficient vector is appropriate.

The approximate process of the algorithm is as follows [13]:

Set the approximate range of speeds so that the speed of the particles can safely avoid certain problems within the speed range and set the initial speed and initial position in the speed interval and search interval. 
The individual's extremism finds the best positional information for each particle, and we find the best solution from the best solution for so many individuals, which is suitable for the global, and with the previous optimal solution. Make corresponding comparisons and choose the best as our current optimal solution.

The status update formula is:

$$
V_{i d}=\omega V_{i d}+C_{1} \cdot \operatorname{random}(0,1) \cdot\left(P_{i d}-X_{i d}\right)+C_{2} \cdot \operatorname{random}(0,1) \cdot\left(P_{g d}-X_{i d}\right)
$$

Here, the use of $\mathrm{W}$ is used to replace the inertial coefficient, $\mathrm{C} 1$ and $\mathrm{C} 2$ is the acceleration constant.

The termination condition is to make algebra $G_{\max }$ to reach maximum [14].

\section{DESIGN EXAMPLES}

When we move our heads slightly, we think of the array of arrays below. Let us call it the five-arm array [15] for the time being. It contains 20 array elements.

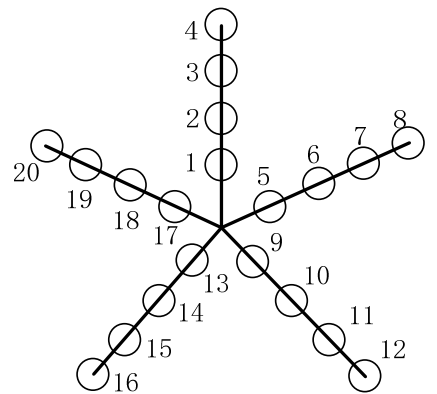

FIGURE 3. Five-arm arrays

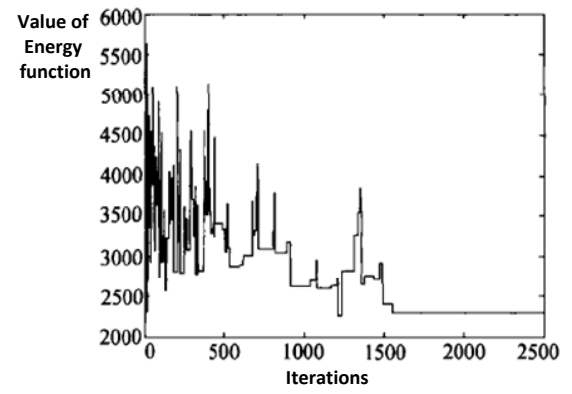

FIGURE 4. Multiple iterations

Assuming that the frequency of the signal is $3000 \mathrm{~Hz}$, the maximum side-lobe of the 21 surrounding beams in the azimuth dimension is not greater than $-28 \mathrm{db}$. The weighted average of each particle gives the regular beam pattern as follows:

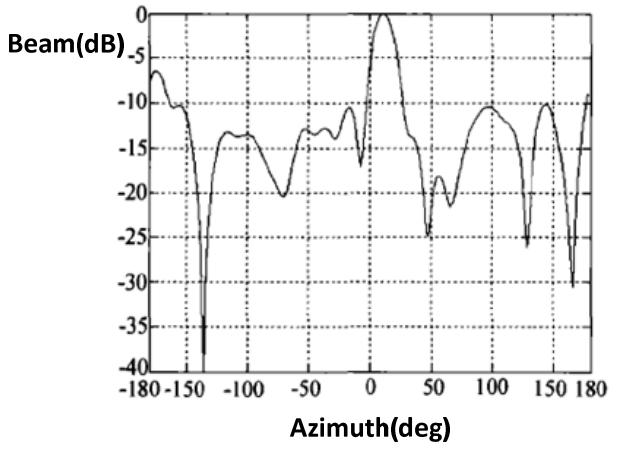

FIGURE 5. The regular beam pattern

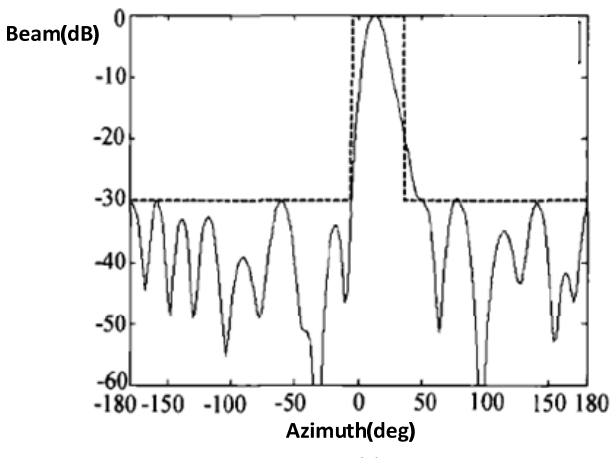

FIGURE 6. Improved beam pattern

Now we use the algorithm of particle swarm described earlier to simulate the following improved beam pattern:

It can be seen that the side-lobe of the beam pattern after processing by the particle swarm algorithm becomes lower, which is exactly what we want, and the convergence of the algorithm can be obtained through multiple iterations. See the figure below: 


\section{CONCLUSION}

This paper presents an intelligent algorithm for the disadvantage of high side lobe caused by conventional beamforming, namely, the particle swarm algorithm. The simulation experiments verify that this algorithm is more effective. We have established the concept of a cost function so that we can quantitatively give the result to be sought. It should be noted that for different array structures, the cost function should be reasonably selected when using this method in order to make the algorithm converge as quickly as possible or achieve better performance.

\section{REFERENCES}

1. Kennedy J, Eberhatl R C Particle Swarm Optimization. Proc. IEEE International Conference on Neural Networks.V01.2:1942-1948.

2. Dolph,C.L "A Current Distribution of Broadside Arrays which Optimize the Relationship between beam width and side-lobe level',,Proc.Inst.RadioEngsr,34,pp.335,June(1046).

3. Pritehard, R. L "Optimum Directivity Patterns for Linear Point Arrays", J.Aeoust.Soe.Am.,26, (1954), PP.1034.

4. Angeline P J Evolutionary Optimization Versus Particle Swarm Optimization: Philosophy and Performance Differences. Proc. Seventll Annual Conference on Evolutionary Programming,1998,256-260.

5. Davids N, Thurston E G, Mueser R E.J. Acous. Soc. Am., 1951,24(1):50 56.

6. EL Gamal A. Using Simulated Annealing to Design Good Codes, IEEE Trans IT,1987,33(1):116 123.

7. Rockafellar R.T., Uryasev S. Optimization of Conditional Value-at-risk. The Journal of Risk[J],2000,2(3)21-41.

8. Eberhart R C, Kenned Y J. A New Optimizer Using Particle Swarm Theory/ /Proc. of the 6th International Symposium on Micro Machine and Human Science, Nagoya, Japan,1995.

9. Kassabalidis ISharkawi E I, Marks M A, et al. Adaptive-SDR: Adaptive Swarm-based Distributed Routing/ /Proc. of the 2002 International Joint Conference on Neural Networks,2002.

10. Eberhart RC, Shi Y H. Particle swarm optimization: Development applications and resources. Proc Congress on Evolutionary Computation2001. Piscataway, NJ: IEEE Press,2001: 81 - 86.

11. Kennedy J, Eberhart R C. Particle Swarm Optimizations. Proceedings of IEEE International Conference on Neutral Networks, Perth, Australia,1995. 1942 - 1948.

12. Konno H., Yamazaki H., Mean Absolute Deviation Portfolio Optimization Model and Its Application to Tokyo Stock Market. Management Science[J],1991,37(5)519-531

13. Eberhart R, C E R, Eberhart R C. Comparing inertia weights and constriction factors in particle swarm optimization [C]. //Evolutionary Computation, 2000. Proceedings of the 2000 Congress on. IEEE, 2000:84 88.

14. Angeline P J. Evolutionary optimization versus particle swarm optimization: Philosophy and performance differences [J]. Lecture Notes in Computer Science, 1998:601-610.

15. Van D, F V D B, Van D B F. A Cooperative approach to particle swarm optimization [J]. Volonary Omaon Ranaon on, 2004, 8:225 - 239. 